\title{
Large Spinal Meningioma with Hemorrhage after Selective Root Block in the Thoraco-Lumbar Spine
}

\author{
Heui Seung Lee', Sang Hyung Lee ${ }^{2}$, \\ Young Seob Chung ${ }^{2}$, Hee-Jin Yang ${ }^{2}$, Young-Je Son ${ }^{2}$, Sung Bae Park ${ }^{2}$ \\ ${ }^{l}$ Department of Neurosurgery, Seoul National University Hospital, Seoul, \\ ${ }^{2}$ Department of Neurosurgery, Seoul National University Boramae Medical Center, Seoul, Korea
}

Spinal meningioma accounts for $25 \%$ of all spinal cord tumors and occurs mostly in the thoracic region. Spontaneous intraspinal bleeding associated with spinal meningioma has rarely been reported. Most cases of hemorrhage associated with meningiomas are extratumoral and subarachnoid, whereas subdural and intratumoral hemorrhage cases have been reported to occur rarely. We experienced a case of a 58-year-old woman with thoracolumbar spinal meningioma accompanied by intraspinal subdural hematoma. She presented with progressively worsened back pain and newly developed weakness in the right lower extremity after a selective nerve root block in the lumbar spine. She underwent the operation and progressively showed neurological recovery during the postoperative course. We report a thoracolumbarspinal meningioma with subdural and intratumoral hemorrhage after a spinal procedure that caused a sudden neurological deterioration.

Key Words: Spinal meningioma $\cdot$ Hemorrhage

\section{INTRODUCTION}

Relatively rare compared to its intracranial counterpart, spinal meningioma accounts for $1.2 \%$ of all meningiomas of the central nervous system and $25 \%$ of all spinal cord tumors ${ }^{6}$. Spontaneous intraspinal bleeding associated with spinal meningioma has rarely been reported, whereas spinal subdural hematoma has occasionally been reported in patients with bleeding diathesis and spinal injury, even after lumbar puncture ${ }^{5,9)}$.

We report a rare case of a 58-year-old woman with thoracolumbar spinal meningioma accompanied by intraspinal subdural hematoma that developed after a spinal nerve block.

\section{CASE REPORT}

A 58-year-old woman was admitted to the hospital with

- Received: October 27, 2013 • Revised: December 3, 2013

- Accepted: December 6, 2013

Corresponding Author: Sung Bae Park, MD

Department of Neurosurgery, Seoul National University Boramae Medical

Center, 39 Boramae Road, Dongjak-gu, Seoul 156-707, Korea

Tel: +82-2-870-3301, Fax: +82-2-870-3863

E-mail: ddolbae01@naver.com

@This is an Open Access article distributed under the terms of the Creative

Commons Attribution Non-Commercial License (http://creativecommons.org/ licenses/by-nc/3.0/) which permits unrestricted non-commercial use, distribution, and reproduction in any medium, provided the original work is properly cited. severe back pain accompanied with nausea and vomiting. Because of the back pain, she underwent a selective nerve root block in the lumbar spine at another hospital. After the spinal procedure, the patient felt more severe back pain, newly developed pain, and weakness in her right leg. She had intact voiding sensation and no difficulty voiding at the time of admission. The paraparesis was checked during a neurologic examination. In the right lower extremity, ankle dorsiflexion (Gr $\mathrm{III} / \mathrm{IV}+)$ and toe flexion and extension (IV-/IV+) weaknesses were prominent compared with those of the left lower extremity. Deep tendon reflex was absent on the right knee, and the "extensor toe sign" was negative bilaterally, without clonus. Sensory impairment to the pain was noted on the L5 dermatome of the right calf.

An MRI revealed a ventrally located intradural mass extending from T10 to L2; the mass was iso- to hypointense on T1-, and the hyperintense portion on the T2-weighted images was compatible to the bleeding inside the tumor. The lesion was heterogeneously enhanced with contrast administration (Fig. 1).

One day after her admission, the patient experienced worsening of her neurological status with weakness of the lower extremities and the loss of anal tone and voiding sensation. She underwent an emergency operation with high-dose methylprednisolone therapy.

A laminectomy from the T10 to L2 levels was performed using a posterior midline approach. After the dura was opened, 

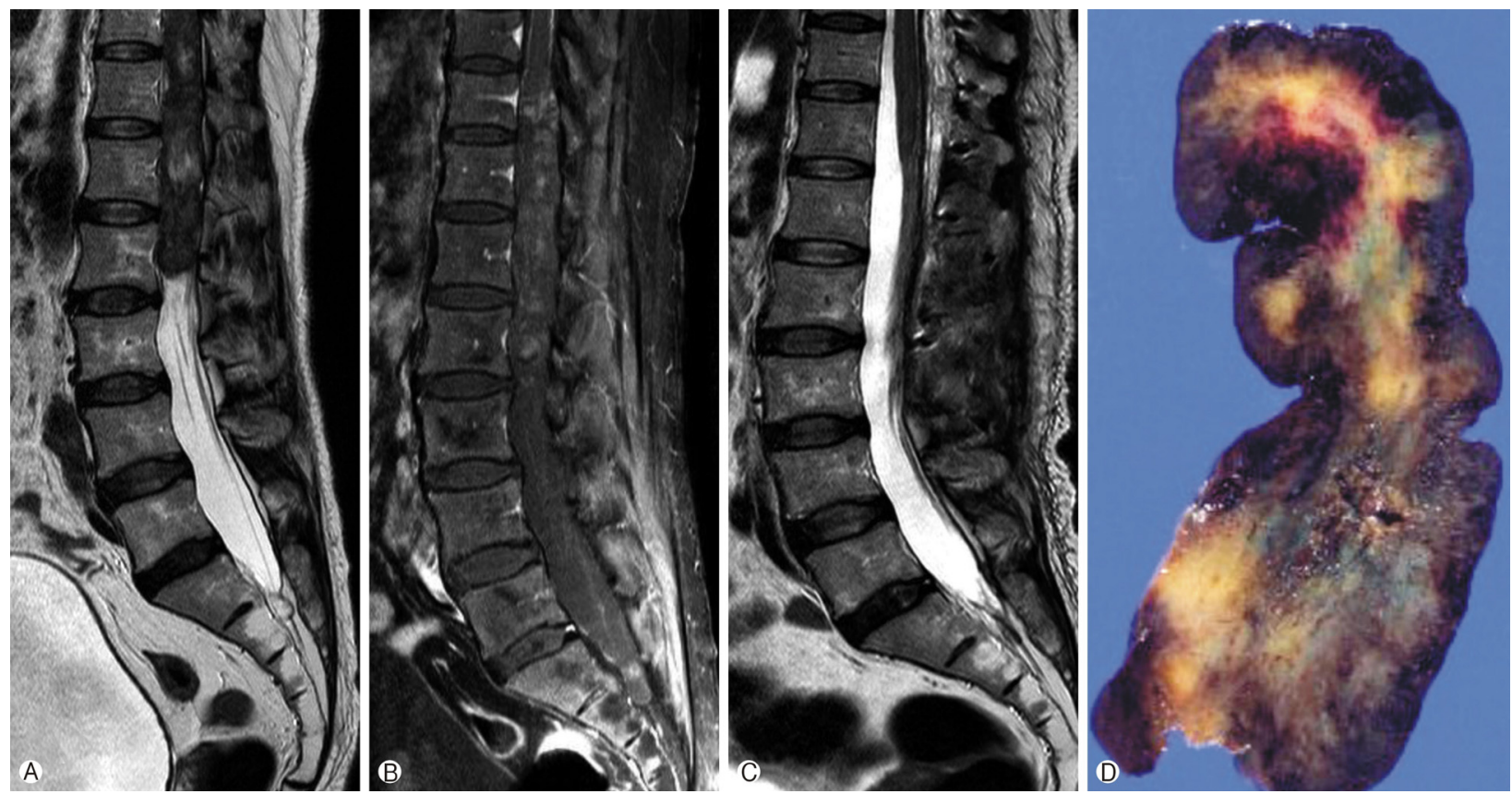

Fig. 1. Preoperative T2-weighted MRI (A) and T1-weighted MRI with gadolinium enhancement (B) revealed the multifocal heterogeneous enhanced intraspinal mass in the thoraco-lumbar area. There was no intraspinal mass in the postoperative T2-weighted MRI (C). Photograph of mass specimen shows that fibrous capsule covered the pale yellow colored core including hemorrhage.
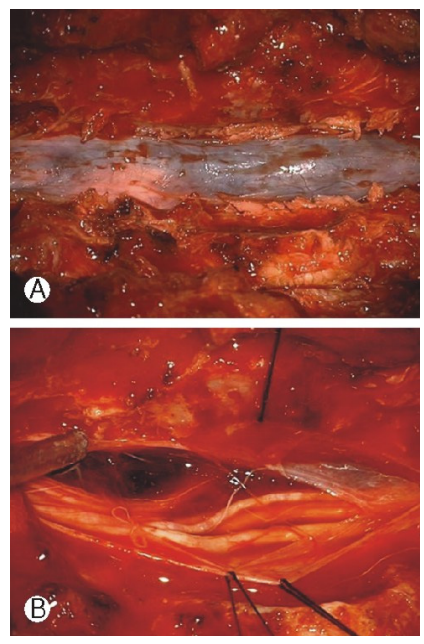

Fig. 2. Intraoperative photography shows the exposed dura after the laminectomy (A), (B) a subdural hematoma after the dura incision and a hemorrhagic intradural mass.

the mass was exposed to be dark and oil-colored, and a chocolate-colored subdural hematoma was observed above and below the mass (Fig. 2). The mass was not connected to the nerve roots. However the mass was adhered to the ventral dura. Therefore, the mass was removed en bloc under an operating microscope, with coagulation of the dural attachment. A frozen section biopsy of the mass was performed, and under microscopic examination, it was compatible with a meningioma. The dura was closed in a watertight fashion with bleeding control. Although no wave was observed on intraoperative monitoring at the beginning of the surgery, some wave was observed at the end of surgery. The pathologic report confirmed the meningioma was a fibrous type WHO grade I/III tumor.

During the postoperative course, the status of motor and sensory was improved. The patient felt bladder fullness and anal contraction after 2 postoperative days. She was transferred to the department of rehabilitation for an intense rehabilitation program. Two months post-surgery, the patient was freed from the Foley catheter, and her urinary symptoms recovered without urinary incontinence, frequency, residual urine sensation, and urgency.

\section{DISCUSSION}

Intraduralextramedullary spinal cord tumors are predominantly meningiomas or peripheral nerve sheath tumors, whereas less common occurrences include paragangliomas, metastases, lipomas, spinal nerve sheath myxomas, sarcomas, and vascular tumors ${ }^{1)}$. Meningiomas are dural-based tumors that arise from arachnoid cap cells and, consequently, can be found in any location that dura is present. Approximately $25 \%$ of all primary spinal cord tumors are meningiomas. More than $80 \%$ of spinal cord meningioma patients are women, and $80 \%$ of these tumors occur in the thoracic region. In men, spinal cord meningiomas are equally distributed between the cervical and thoracic cords. Overall, $15 \%$ of spinal cord meningiomas occur 
in the cervical spine, $81 \%$ in the thoracic spine, and $4 \%$ in the lumbar spine. Most meningiomas are slow-growing lowgrade tumors ( $\mathrm{WHO}$ grade I $)^{1,4)}$.

The clinical symptoms of spinal meningioma depend on the tumor location, with respect to the spinal cord and nerve roots. Common presenting symptoms include back pain (70\%), motor weakness (60\%), sensory change (40\%), and incontinence $(40 \%)^{1,7}$.

Acute subdural spinal hematoma has been reported to occur rarely; however, after it occurs, neurological deficits caused by cord compression from a hematoma may occur. The typical clinical presentations of acute subdural spinal hematoma are the sudden onset of spinal or radicular pain and myelopathy, including paraplegia and sensory and autonomic dysfunction. An MRI scan is quite useful for the early detection of the hemorrhage and for establishing the correct diagnosis. Nontraumatic acute spinal subdural hematomas have been reported in cases of coagulation abnormalities and iatrogenic causes, such as spinal punctures. Swann reported that spinal tumors, particularly in the region of the caudaequina and conus medullaris, can cause spontaneous spinal subarachnoid hemorrhage, although this occurrence is uncommon. The mechanism of hematoma formation is believed to be related to the vulnerability to trauma of the blood vessels surrounding these tumors $^{8)}$. Most cases of hemorrhage associated with meningiomas are extratumoral and subarachnoid, whereas subdural, intracerebral and intratumoral hemorrhage cases are reported less frequently ${ }^{2,5,10)}$. In our case, the patient was considered to have subdural and intratumoral hemorrhages after an epidural block that most likely caused the subdural space injury. The injected particle into tissue including vascular structures around tumor could induced the hemodynamic changes and the hemodynamic changes may cause the intratumoral hemorrhage.

In cases in which an acute subdural hematoma is formed either from iatrogenic or coagulation abnormalities, early surgical treatment is always important for the prognosis when the patient's neurological status progressively deteriorates ${ }^{2}$. The treatment of spinal meningiomas is predominantly surgical. A recent study that analyzed 131 patients who underwent surgical resection of a spinal meningioma found that the spinal meningioma complete resection rate was $97 \%$, and the procedure was generally considered to be effective following bipolar coagulation $^{6}$.

When the neurological status of a patient is not complica- ted, favorable outcomes are achieved in most cases. However, progressive spinal cord compression by spinal meningioma with neurologic deterioration may result in permanent neurologic deficit even after successful surgery ${ }^{7}$. The long-term functional outcome of spinal meningioma treatment has been reported to be correlated with the neurological deficit severity at the time of presentation and the symptomduration ${ }^{3)}$.

\section{CONCLUSION}

Although spinal meningioma with bleeding has been reported, reports of spinal meningioma associated with iatrogenic causes, such as spinal nerve block, are rare to the best of our best knowledge. If a severe neurological deterioration occurs after a spinal procedure, an intra-spinal mass with hemorrhage could be considered.

\section{REFERENCES}

1. Chamberlain MC, Tredway TL: Adult primary intradural spinal cord tumors: a review. Curr Neurol Neurosci Rep 11:320-328, 2011

2. Domenicucci M, Ramieri A, Ciappetta P, Delfini R: Nontraumatic acute spinal subdural hematoma: report of five cases and review of the literature. J Neurosurg 91(1 Suppl):65-73, 1999

3. Haegelen C, Morandi X, Rifaud L, Amilashi SF, Leray E, Brasier G: Results of spinal meningioma surgery in patients with severe preoperative neurological deficits. Eur Spine J 14:440-444, 2005

4. Mechtler LL, Nandigam K: Spinal cord tumors: new views and future directions. Neurol Clin 31:241-268, 2013

5. Morimoto T, Tokunaga H, Hoshida T, Tsunoda S, Sakaki T: Thoracic meningioma presenting subarachnoid haemorrhage. Acta Neurochir (Wien) 138:886-887, 1996

6. Sandalcioglu IE, Hunold A, Müller O, Bassiouni H, Stolke D, Asgari S:Spinal meningiomas: critical review of 131 surgically treated patients. Eur Spine J 17:1035-1041, 2008

7. Subačiūtė J: Spinal meningioma surgery: predictive factors of outcome. Acta Med Lituanica 17:133-136, 2010

8. Swann KW: Spontaneous spinal subarachnoid hemorrhage and subdural hematoma: report of two case. J Neurosurg 61:975-980, 1984

9. Toledo E, Shalit MN, Segal R: Spinal subdural hematoma associated with anticoagulant therapy in a patient with spinal meningioma. Neurosurgery 8:600-603, 1981

10. Vij M, Jaiswal S, Jaiswal AK, Kumar S, Behari S: Meningioma with hemorrhagic onset: two case reports. J Can Res Ther 8: 145-147, 2012 